\title{
On the Reasonable Period Involved in Article 95 of Chinese Contract Law
}

\author{
Lijuan Zhang ${ }^{1}$, Yanwei Wang ${ }^{2}$, Jie Zhao ${ }^{3}$ \\ ${ }^{1)}$ School of economics and management, Northeast Dianli University, Jilin, Jilin, China (zhanglijuannedu@163.com) \\ ${ }^{2)}$ School of economics and management, Northeast Dianli University, Jilin, Jilin, China (wangyanwei@nedu.edu.cn) \\ ${ }^{3)}$ School of law, Jilin University, Changchun, Jilin, China (626287052@qq.com)
}

\begin{abstract}
Although there is a provision about the period to exercise the right to terminate a contract in Article 95 of Chinese Contract law, it is not concrete. This paper analyze the reasonable period under the situation that the party urge or not and then give some practical advices to the parties of the contract.
\end{abstract}

Keywords—right to cancel a contract, reasonable period, identify

\section{刍议我国合同法第 95 条中的合理期限}

\author{
张丽娟 ${ }^{1}$ 王延伟 $^{2}$ 赵洁 $^{3}$ \\ 1) 东北电力大学经济管理学院, 吉林, 吉林, 中国 \\ 2) 东北电力大学经济管理学院, 吉林, 吉林, 中国 \\ 3) 吉林大学法学院, 长春, 吉林, 中国
}

摘＼cjkstart要 我国合同法第 95 条对合同解除权的行使期间做出了规定，然而在法律没有规定或者当事人没有约定解除权行使期限的 情况下, 对方当事人在何时不行使催告权, 解除权人的解除权消灭却未做出明确规定。本文区分当事人是否进行催告两种情形, 对该 合理期限的认定提出若干意见。

关键词 合同解除权, 合理期限, 认定

1. 引言

我国《合同法》第 95 条规定: “法律规定或者当事人 约定解除权行使期限, 期限届满当事人不行使的该权利消 灭。法律没有规定或者当事人没有约定解除权行使期限, 经对方催告后在合理期限内不行使的, 该权利消灭。” 该 条文仅就法律规定或者当事人约定解除权行使期限情况下 的合同解除权存续期间做出了相应规定, 而在法律未规定 且当事人也无约定的情况下, 对方催告后的合理期限应为 多长? 在上述情形下, 非解除权人催告时能否为解除权人 指定一个逾期不行使解除权, 解除权即消灭的行使解除权 的期限? 当事人在合理期限内若未行使催告权, 合同解除 权是否消灭? 该合理期限应该如何认定? 本文仅就以上几 点问题提出若干想法。

\section{2. 经当事人催告之情形}

\section{1 法无明文规定及当事人无约定之情形}

在法律无规定, 当事人也无约定的情况下, 合同法并 未明确规定当事人催告后合理期限的长短, 不能说这不是 一个立法缺陷。《最高人民法院关于审理商品房买卖合同纠 纷案件适用法律若干问题的解释》(以下简称为法释[2003]7 号司法解释) 的规定。该解释第十五条第二款规定: “法律 没有规定或者当事人没有约定，经对方当事人催告后，解 除权行使的合理期限为三个月。对方当事人没有催告的, 解除权应当在解除权发生之日起一年内行使; 逾期不行使 的, 解除权消灭。” 该解释明确规定了在当事人催告与否前 提下的解除权的存续期间, 即分别为三个月和一年, 这为 解决其它合同的类似问题提供了一定的借鉴。那么, 该规 定可否类推适用于其他类型的合同呢? 笔者以为, 催告后 
的合理期限属于一种除斥期间, 即法律规定的某种权利的 预定存续期间, 权利人于除斥期间内不行使权利的, 则丧 失该权利。解除权的行使是一个十分严肃的问题, 因而法 律或司法解释明确规定解除权的除斥期间是非常有必要 的。然而, 在当事人未约定、法律也无规定的情况下, 如 何确定该合理期限的确是一个十分棘手的问题。法释 [2003]7 号司法解释对商品房买卖合同的除斥期间做出了 规定, 如仅从学理上探讨, 把适用于某一特定领域的司法 解释所体现出来的原理, 扩张到整个合同法领域也并无不 妥。那么在当事人催告的情形下, 合理期限可以参照法释 [2003]7 号司法解释的规定确定为三个月。

\section{2 非解除权人能否指定行使解除权的期限之情形}

《合同法》第九十五条的一个重要的欠缺就是未规定 非解除权人催告时能否为解除权人指定一个解除权人行使 解除权的期限, 逾期不行使解除权的, 解除权即消灭。笔 者认为, 催告权是平衡违约方与守约方利益的一种权利, 违约方催告的目的就是希望因自身的违约行为而产生的不 确定的社会经济关系能够尽快稳定, 而此处的尽快除与交 易性质、违约原因、交易标的情况等有关外, 还与交易主 体的具体状况有关系, 不能一概而论。但无论怎样, 从催 告权的本意来看, 在当事人催告的情形下, 非解除权人可 以为解除权人指定行使解除权的期限, 逾期不行使解除权 的, 解除权即消灭。德国民法典、日本民法典中也均明确 规定另一方当事人可以为权利人指定行使的适当期间, 解 除权人不在此期间届满前表示的, 解除权消灭。

\section{3. 未经当事人催告之情形}

法律若无规定, 当事人也无约定, 非解除权人又不进 行催告, 非但上述状态不能结束, 解除权人还将从中获取 不恰当的利益。对于该合理期限笔者有如下看法。

\section{1 合理期限期间之总体原则}

合理期限这一除斥期间不宜过长。行使解除权实际上 是对违约方不履行到期债务的一种较为严厉的惩罚, 其后 果是导致双方合同法律关系终止, 财产关系恢复原状或终 止履行。而债权的一般诉讼时效为两年, 为了防止债权诉
讼时效过期而债权人还掌握着较为严厉的解除权情形的发 生, 解除权的行使期限应短于作为承担一般违约责任的诉 讼时效之两年的时间, 否则对债务人将明显不利, 也会对 诉讼时效制度造成冲击, 导致合同关系极不稳定, 这与法 律平衡双方当事人权利的公平理念及价值观也是相违背 的。

\section{2 合理期限期间之具体设计}

司法实践中可将合同解除权的除斥期间确定为一年。

原因有二: 一是合同解除权属于形成权, 我国合同法 关于形成权除斥期间（如撤销权）的规定一般为一年，从 立法传统和守法习惯上, 一年的时间是可取的; 二是法释 [2003]7 号司法解释中也规定: “对方当事人没有催告的, 解除权应当在解除权发生之日起一年内行使; 逾期不行使 的, 解除权消灭。” 基于上述经当事人催告之情形中的相关 论述, 可以参照该司法解释将此种情况下合同解除权的除 斥期间确定为一年。

综上, 由于合同解除条件成就时, 解除权人可以随时 结束合同, 当解除权人急于行使解除权时, 为了避免使双 方当事人之间的权利义务关系长期处于不稳定的状态下， 合同相对人可以在以上合理期限内行使催告权, 以便更好 地实现双方的利益。

\section{参考文献(References)}

[1] H. H. Shi, "On Cancel a Contract and Its Restrict," Legal System and Society, no. 5, pp. 21-22, 2014.

[2] Y. F. Jiang, "On the Exercise of Right to Terminate a Contract and its Legal Consequence," Journal of Changshu Institute of Technology, no. 6, pp. 74-76, 2008.

[3] W. C. Xue, "Cancel a Contract and Exercise of Right to Cancel the Contract," Oriental Law, no. 1, pp. 152-160, 2008.

[4] Z. L. Wang and K. Du, "On Exercise of the Right to Cancel a Contract," Journal of SWUPL, vol. 7, no. 1, pp. 105-109, 2005.

[5] L. L. Liu, "On the Exercise of Right to Terminate a Contract," Wuhan University of Technology (Social Science Edition), vol. 17, no. 6, pp. 757-760, 2004. 